when $\mathrm{CpG}$ suppression is absent in a $\mathbf{G}+\mathbf{C}$ rich region in accordance with the tight helix model, the germ-line undermethylation model is not excluded. Thus in bovine satellite I DNA, where $\mathrm{CpG}$ suppression is essentially absent and $G+C$ is high (about 57\%), genomic sequencing experiments have indicated that these sequences are undermethylated in sperm but not in somatic (thymus) DNA (J. Herbert Taylor, personal communication).

Therefore I believe that, while the tight helix model offers an attractive alternative that may explain some $\mathrm{CpG}$-rich regions, available data do not warrant abandonment of the undermethylation model. To test the undermethylation model, we are planning experiments that may reveal the methylation status of some CpG-rich regions of MHC genes in sperm DNA; we do not presently endorse any particular prediction for the results of these experiments. Even if further data should favour the tight helix model as a mechanistic explanation for the maintenance of $\mathrm{CpG}$-richness, the conservation of this feature in several MHC genes across three species suggests some functional significance (of either CpG-richness or $\mathrm{G}+\mathrm{C}$-richness) that remains to be explained.

National Cancer Institute,

EDWARD E. MAX

National Institutes of Health,

Bethesda,

Maryland 20205, USA

1. Max, E.E. Nature 310, 100 (1984).

2. Tykocinski, M.L. \& Max, E.E. Nucleic Acids Res. 12, 4385 (1984).

3. Erickson, R.P. Nature 311, 217 (1984)

4. Adams, R.L.P. \& Eason, R. Nature 312, 407 (1984)

5. Rahe, B., Erickson, R.P. \& Quinto, M. Nucleic Acids Res. 11, 7947 (1983).

6. Goddard, J.P. \& Schulman, L.H. J. biol. Chem. 247, 3864 (1972).

7. Shapiro, R. et al. J. biol. Chem. 248, 4060 (1973).

8. Horz, W. \& Altenberger, W. Nucleic Acids Res. 9, 683 (1981).

9. Ponzetto-Zimmerman, C. \& Wolgemuth, D.J. Nucleic Acids Res. 12, 2807 (1984).

10. Sanford, J., Forrester, L. \& Chapman, V. Nucleic Acids Res. 12, 2823 (1984).

\section{In search of \\ physical reality}

SIR - T.W. Marshall's review ${ }^{1}$ of my book In Search of Reality calls for some serious factual qualifications.

The question at issue concerns (in Marshall's words) the possibility or impossibility of physical theories of a "local realist type" essentially built along the lines of Einstein's physical realism (as is well known, Einstein's locality requirements were an essential part of his criticism of the Copenhagen philosophy). Marshall expresses the view - presented by him as certain - that even within the domain of such theories there is "no difficulty at all in explaining the data obtained in the experiments so far performed"', provided only that determinism is not imposed and that the domain of the theories in question is not arbitrarily restricted to models picked up within too narrow a range.

This claim is highly misleading in that it ignores the main result of the Bell theorem, namely, that Einstein's local realism is incompatible with some elementary and in principle verifiable predictions of quantum mechanics, quite independently both of models (deterministic or indeterministic) and of any actual experimental result (proofs such as the one transcribed in plain words in the book are, despite superficial appearances, fully general in this respect). Marshall's claim is even plainly erroneous since, when they turn their attention to the results of the experiments so far performed in this field (by Clauser, Fry, Aspect and others), realist physicists (including those who sympathize most with the Einsteinian ideals) generally stress that conceptually it is, by now, if not logically impossible, at least extremely difficult to preserve Einstein's local realism; for, they say, it seems hopeless to do so without attributing the agreement found between the experimental data and the quantum predictions to some coincidence; and this, on the other hand, seems quite unbelievable in view of the quality of the agreement in question. In comparisons of this kind, the inefficiency of the counters is extremely important, but, contrary to a suggestion by Marshall, it does not by itself remove the difficulty at issue - see, for example, refs 2 and 3 for detailed quantitative data and evaluations.

Here and there, various more-or-less convincing attempts are now being made to build up realistic nonlocal theories. But it can safely be asserted that a plain regression to the kind of local physical realism that Einstein so strongly advocated is ruled out.

\section{Laboratoire de Physique Théorique et}

Particules Elémentaires,

\section{Centre d'Orsay,}

\section{Orsay, France}

1. Marshall, T.W. Nature 308, 669 (1984)

2. Clauser, J.F. \& Shimony, A. Rep. Prog. Phys. 41, 1881-1927 (1978).

3. Aspect, A., Grangier, P. \& Roger. G. Phys. Rev. Lett. 49, 91-94 (1982).

T.W. Marshall RePlies - Bernard d'Espagnat says it is "extremely difficult if not logically impossible" to construct a local realist explanation for the results of photon-correlation experiments of the Aspect type, while I, in my review of his book In Search of Reality, claimed that it is "not at all difficult". I have reached what may seem to be the opposite conclusion from d'Espagnat as a result of reading precisely the literature he cites above in support of his view.

How is that possible?

First we should dispose of the suggestion of "logical impossibility". What d'Espagnat is saying here is that Bell's theorem demonstrates that an ideal experiment, with perfect detectors, will give results either corroborating the quantum theory of measurement and refuting all local realist theories or the oppposite. In the absence of such an ideal experiment, the assessment of real experiments, such as those of Aspect, becomes one of distinguishing between "extremely difficult" and "not at all difficult", which shows that d'Espagnat and I have made opposite value judgements.

The review article of Clauser and Shimony ${ }^{1}$ makes it quite clear what is at stake here. We can discriminate between the quantum model and "the family of local realist models" with an experiment using low-efficiency detectors, but only if we restrict the latter by imposing an additional restriction called "no enhancement". From the Aspect experiment, we might claim that local realist models satisfying the no-enhancement restriction are no longer possible. (Even this claim is, however, over-ambitious, because the procedure by which a rather weak "signal" is extracted from a rather large "noise" background is open to criticism ${ }^{2}$.)

What is this no-enchancement restriction? Essentially it imposes on all conceivable local realist theories certain features of the quantum model: that light is transmitted as photons and that the successive interactions of a photon with first a polarizing device and then a photomultiplier are statistically independent. By dropping these assumptions, my colleagues Franco Selleri, Emilio Santos and I have been able, without difficulty, to construct several local realist models ${ }^{3-5}$, giving coincidence counts which are consistent with Aspect's results ${ }^{6}$. The imposition of the noenhancement restriction begs the question in favour of the quantum model, much as the von Neumann "theorem" did at an earlier stage in the debate.

If anyone suspects that this is an "angels on a pinhead" type of controversy, it should be noted that our criticism of the data analysis in existing experiments has shown us the correct way to analyse the coincidence data from low-efficiency detectors ${ }^{7}$. This research indicates that a discrimination between the quantum model and a much wider family of local realist models, not subject to the no-enhancement restruction, is possible.

In the meantime I think that most scientists, given a choice between abandoning physical reality and abandoning such an ad hoc hypothesis as "no enhancement", will unhesitatingly choose the latter course. This must mean conceding that Einstein's criticism of the Copenhagen philosophy remains valid for some of us, overwhelmingly so.

T. W. MARSHALL

Department of Mathematics,

Manchester University,

Manchester M13 9PL, UK

1. Clauser, J.I. \& Shimony, A. Rep. Phys. 41, 1881-1927 (1978) . Marshall, T.W., Santos, E. \& Selleri, F. Lett. Nuovo Cimento 38, 417 (1983).

Marshall, T.W., Santos, E. \& Selleri, F. Phys. Lett. 98A 5 (1983).

4. Marshall, T.W. Phys. Lett. 99A, 163 (1983).

5. Garuccio, A. \& Selleri, F. Phys. Lett. (in the press).

5. Garuccio, A. \& Selleri, F. Phys. Lett. (in the press). 91-94 (1982).

Marshall, T.W. Phys. Lett. 100A, 225 (1984) 